\title{
Scalar field induced oscillations of neutron stars and gravitational collapse
}

\author{
Florian Siebel(1), José A. Font ${ }^{(1)}$ and Philippos Papadopoulos ${ }^{(2)}$ \\ ${ }^{(1)}$ Max-Planck-Institut für Astrophysik, Karl-Schwarzschild-Str. 1 \\ D-85741 Garching, Germany \\ ${ }^{(2)}$ School of Computer Science and Mathematics, University of Portsmouth \\ PO1 2EG, Portsmouth, United Kingdom
}

(June 10, 2018)

\begin{abstract}
We study the interaction of massless scalar fields with self-gravitating neutron stars by means of fully dynamic numerical simulations of the Einstein-Klein-Gordon perfect fluid system. Our investigation is restricted to spherical symmetry and the neutron stars are approximated by relativistic polytropes. Studying the nonlinear dynamics of isolated neutron stars is very effectively performed within the characteristic formulation of general relativity, in which the spacetime is foliated by a family of outgoing light cones. We are able to compactify the entire spacetime on a computational grid and simultaneously impose natural radiative boundary conditions and extract accurate radiative signals. We study the transfer of energy from the scalar field to the fluid star. We find, in particular, that depending on the compactness of the neutron star model, the scalar wave forces the neutron star either to oscillate in its radial modes of pulsation or to undergo gravitational collapse to a black hole on a dynamical timescale. The radiative signal, read off at future null infinity, shows quasi-normal oscillations before the setting of a late time power-law tail.
\end{abstract}

PACS number(s):04.25.Dm, 04.40.-b, 95.30.Lz, 04.40.Dg, 97.60.Lf

\section{INTRODUCTION}

Obtaining reliable estimates for gravitational wave signals emitted from the stellar collapse of massive stars is one of the key motivations for numerical relativity. The strength of actual astrophysical sources is still under investigation, as the relevance of such sources for the first and second generation of interferometric detectors depends on the details of signal amplitude and frequency, but also on the occurrence rates (for a current view, see [i]). In any case, relativistic collapse is a fundamental physical process, and the development of relevant computational procedures has been a long steady process over the past three decades (see, e.g. [2 [4]; see also [5] and references therein). In contrast to a Newtonian approximation, where the computational problem is well defined and attention can be devoted to astrophysical details [5.,6], there is no consensus as to what is the optimal, or at least adequate, framework for developing relativistic simulations.

We will use here the so-called characteristic formulation of general relativity [7, 8 . The formalism has been developed specifically for addressing ambiguities concerning gravitational radiation and is well adapted to handle the propagation of signals. It allows for spacetime compactification, which avoids problems due to the artificial reflection of the fields at outer boundaries. In addition, it allows for the extraction of physically relevant global quantities like the News function and the Bondi mass. Nevertheless, the computation of the dynamics of sources of signals is a separate issue altogether, which has not been addressed in this early work. The framework for computing a complete spacetime within the characteristic approach has been laid out in [9] and more explicitly in [10]. The translation of this framework into a compu- tational tool for vacuum spacetimes, in any dimensions, has been a largely successful process (see [11] and references therein).

There are some noteworthy issues. Firstly, the domain of applicability is limited to configurations in which the set of light-cones that forms the basis of the coordinate system does not fold itself into caustics. This puts a limit, for example, on the type of binary systems that can be studied. Secondly, to obtain a complete spacetime one must include in the computational domain the vertex of the light-cones. This involves regularity conditions (and for explicit integration methods, severe timestep restrictions), which at present have been resolved only up to axisymmetric configurations [12]. Nevertheless, the approach has remarkable economy and stability, which makes it a good candidate for studies of isolated relativistic objects emitting gravitational radiation. For the simulation of realistic sources, one would have to include suitable matter models. It was demonstrated in 13. that the modern techniques of high-resolution shockcapturing schemes for solving the equations of relativistic fluid dynamics can be effectively integrated within this framework. A separate study implementing the approach focused on the gravitational radiation properties of an accreting black hole [14.

This work is the first example of the use of characteristic numerical relativity for the study of dynamical neutron star spacetimes, collapse and radiative signals. The present numerical study is performed in spherical symmetry and uses a self-gravitating, massless, scalar field. The scalar field serves as a simple matter model which mimics gravitational waves. It has been used frequently to study global properties of spacetimes, black hole formation and radiative signals. For the latter, the work focused on the interaction of scalar waves and 
black holes (e.g. [15, 16, and especially on the emergence of power-law tails 17 20, which arise from late time backscattering of the scalar field at the exterior spacetime geometry [21]. There are few studies of the interaction of scalar fields with fluid stellar objects. Recently 22 analyzed the scattering of scalar fields off boson stars and the emergence of critical solutions for this setup. They found that the scalar field can either make the boson star collapse to a black hole or to disperse its mass to infinity.

Time-dependent simulations of scattering of gravitational waves packets with neutron stars, as a means of computing the frequency spectrum of the neutron star (see, e.g. 23 for a recent review), have been studied by Allen et al 24 for polytropic equations of state (EoS), and by Ruoff 25] for more realistic EoS. Such simulations were performed using linear perturbation codes. Pavlidou et al. 26] studied the radiative falloff of scalar fields in neutron star spacetimes, using (idealized) analytic, constant density neutron star models and assuming stationarity for the fluid and the geometry.

In the present work we investigate the nonlinear dynamics of neutron stars interacting with scalar fields. We are especially interested in the following questions: How does a stable neutron star react when it interacts with the scalar field? Can the scalar field force the star to undergo gravitational collapse? What is the result of the interaction on the scalar field? In order to answer these questions we perform spherically symmetric, coupled evolutions of the Einstein-Klein-Gordon perfect fluid system. We study the reflection of finite scalar wave packets off neutron stars for a series of stars parametrized by the central density for a given polytropic EoS with polytropic index $n=1$. Our study focuses on the fate of the system during the interaction, both on the generation of nonlinear oscillations in the neutron star and on the gravitational collapse of the neutron star to a black hole.

The paper is organized as follows: Section II describes the basic mathematical foundations of our approach. In Section III, we briefly discuss the numerical techniques and the implementation used in the simulations. In Section IV, we present several numerical tests of our code, aimed to assess the correct implementation of its different components, the hydrodynamic evolution, the scalar field evolution and the metric solver. Section $\mathrm{V}$ describes the actual numerical investigation on the interaction between the neutron stars and the scalar field. Finally, Section VI summarizes our findings. Throughout the paper we use geometrized units $G=c=1$ and further assume that $M_{\odot}=1$. Greek indices run from 0 to 3 .

\section{MATHEMATICAL FRAMEWORK}

We consider a general spherically symmetric spacetime with a two component stress energy tensor of a perfect fluid and a scalar field, $T^{\mu \nu}=T_{F}^{\mu \nu}+T_{\Phi}^{\mu \nu}$. The geometry of our setup follows the lines of the Tamburino-Winicourformalism [9], in particular as it is applied in regular spacetimes, where the foliation of light-cones emanates from a freely falling central observer [10].

\section{A. Einstein equations}

By adopting the Bondi-Sachs [7, 8] form of the metric element in spherical symmetry,

$d s^{2}=-\frac{e^{2 \beta} V}{r} d u^{2}-2 e^{2 \beta} d u d r+r^{2}\left(d \theta^{2}+\sin \theta^{2} d \phi^{2}\right)$,

the spacetime geometry is completely described by the two functions $\beta(u, r)$ and $V(u, r)$.

A sufficient set of Einstein equations for obtaining the spacetime development are grouped as

$$
\begin{aligned}
G_{u r} & =\kappa T_{u r}, \\
G_{r r} & =\kappa T_{r r}, \\
\left.G_{u u}\right|_{\Gamma} & =\left.\kappa T_{u u}\right|_{\Gamma},
\end{aligned}
$$

where the $u$ coordinate is defined by the level surfaces of a null scalar (i.e., a scalar $u$ satisfying $\nabla^{\mu} u \nabla_{\mu} u=0$ ). The $r$ coordinate is chosen to make the spheres of rotational symmetry have area $4 \pi r^{2}$. The $x^{2}, x^{3}$ coordinates in this geometry are simply taken to be the angular coordinates $(\theta, \phi)$ propagated along the generators of the null hypersurface, i.e., they parameterize the different light rays on the null cone. With our choice of units the constant $\kappa$ is simply $\kappa=8 \pi$. The first two Einstein equations, Eqs. (2) and (3), contain only radial derivatives and are to be integrated along each null surface. The last equation (4) is a conservation condition, satisfied on the vertex of the null cones $\Gamma$ due to the regularity conditions. We choose $\Gamma$ to be a timelike geodesic which coincides with the origin of a neutron star at $r=0$. Equation (3) may be substituted for by the equivalent expression $g^{A B} R_{A B}=8 \pi g^{A B}\left(T_{A B}-g_{A B} T / 2\right)$, where $R_{\mu \nu}$ is the Ricci tensor and the indices $(A, B)$ run over the angular coordinates $x^{2}, x^{3}$.

Using the line element and Eqs. (2) and (3) the $\beta$ and $V$ hypersurface equations are given by

$$
\begin{aligned}
& \beta_{, r}=2 \pi r T_{r r}, \\
& V_{, r}=e^{2 \beta}\left(1-4 \pi r^{2}\left(g^{A B} T_{A B}-T\right)\right) .
\end{aligned}
$$

The comma in the above equations indicates, as usual, partial differentiation. Boundary conditions for $\left(\beta(u)_{\Gamma}, V(u)_{\Gamma}\right)$ needed for the radial integrations are provided by imposing regularity at the origin, where the coordinate system is assumed to be a local Fermi system, leading to

$$
\begin{aligned}
\beta & =O\left(r^{2}\right), \\
V & =r+O\left(r^{3}\right) .
\end{aligned}
$$


By imposing such condition at the origin, the lapse of coordinate time $d u$ is related to the corresponding lapse of "retarded time" $d \tau$ measured by distant observers at $r \rightarrow \infty$ as

$$
d \tau=e^{2 H} d u
$$

where

$$
H=\lim _{r \rightarrow \infty} \beta
$$

\section{B. Scalar Field equations}

The dynamics of a scalar field $\Phi$ is governed by the minimally coupled Klein-Gordon equation in spherical symmetry,

$$
\nabla_{\mu} \nabla^{\mu} \Phi=0
$$

where $\nabla_{\mu}$ is the covariant derivative. This is the equation of motion for the Lagrangian

$$
L=-\frac{1}{2} \nabla_{\mu} \Phi \nabla^{\mu} \Phi
$$

with a corresponding stress energy tensor given by

$$
T_{\Phi}^{\mu \nu}=\nabla^{\mu} \Phi \nabla^{\nu} \Phi+L g^{\mu \nu} .
$$

Using a characteristic foliation the scalar wave equation, Eq. (11), takes the form

$$
2\left(r \Phi_{, u}\right)_{, r}=\frac{1}{r}\left(r V \Phi_{, r}\right)_{, r} .
$$

In terms of the intrinsic 2-metric of the $(u, r)$ submanifold,

$$
\eta_{C D} d x^{C} d x^{D}=-e^{2 \beta} d u\left(\frac{V}{r} d u+2 d r\right)
$$

where the indices $(C, D)$ run over the coordinates $(u, r)$, Eq. (14) reduces to

$$
\square^{(2)} g=\frac{e^{-2 \beta} g}{r}\left(\frac{V}{r}\right)_{, r},
$$

where $g=r \Phi$ and $\square^{(2)}$ is the D'Alembertian operator associated with $\eta_{C D}$.

\section{Hydrodynamic equations}

The evolution of the fluid is determined by the local conservation laws of stress energy and density current

$$
\begin{aligned}
\nabla_{\mu} T_{F}^{\mu \nu} & =0, \\
\nabla_{\mu}\left(\rho u^{\mu}\right) & =0,
\end{aligned}
$$

where $T_{F}^{\mu \nu}$ is the stress energy tensor of a perfect fluid

$$
T_{F}^{\mu \nu}=\rho h u^{\mu} u^{\nu}+p g^{\mu \nu} .
$$

All quantities in the above expression have their usual meanings: $\rho$ is the mass density, $h=1+\varepsilon+p / \rho$ is the specific enthalpy, $\varepsilon$ is the specific internal energy and $p$ is the pressure of the fluid. Moreover, $u^{\mu}$ is the four-velocity which satisfies the normalization condition $g_{\mu \nu} u^{\mu} u^{\nu}=$ -1 .

Following [13], after introducing the definitions $D=$ $\rho u^{0}, S^{r}=T_{F}^{0 r}$ and $E=T_{F}^{00}$, the fluid equations can be cast into a first-order flux-conservative, hyperbolic system for the state-vector $\mathbf{U}=\left(D, S^{r}, E\right)$ :

$$
\begin{aligned}
D_{, u}+F_{, r}^{r 0}= & -(\ln \sqrt{-g})_{, u} D \\
& -(\ln \sqrt{-g})_{, r} F^{r 0}, \\
S_{, u}^{r}+F_{, r}^{r 1}= & -(\ln \sqrt{-g})_{, u} S^{r} \\
& -(\ln \sqrt{-g})_{, r} F^{r 1}-\Gamma_{\mu \nu}^{r} T_{F}^{\mu \nu}, \\
E_{, u}+F_{, r}^{r 4}= & -(\ln \sqrt{-g})_{, u} E \\
& -(\ln \sqrt{-g})_{, r} F^{r 4}-\Gamma_{\mu \nu}^{u} T_{F}^{\mu \nu},
\end{aligned}
$$

where $\sqrt{-g}=r^{2} \sin \theta e^{2 \beta}$ is the four dimensional volume element and $\Gamma_{\mu \nu}^{\alpha}$ are the Christoffel symbols. The precise form of the vector of fluxes $\mathbf{F}$ can be obtained by using Eqs. (17)-(18) (see also [13]). The explicit relations between the primitive variables $\mathbf{w}=\left(\rho, \varepsilon, u^{r}\right)$ and the conserved variables $\mathbf{U}=\left(D, S^{r}, E\right)$, for a perfect fluid EoS, $p=(\Gamma-1) \rho \varepsilon$, where $\Gamma$ is the adiabatic index of the fluid, are given in 13 .

With the above definitions, the metric equations (5)(6) read, for the combined stress energy tensor of a fluidscalar field system,

$$
\begin{aligned}
& \beta_{, r}=2 \pi r\left(\rho h\left(u_{r}\right)^{2}+\left(\Phi_{r}\right)^{2}\right), \\
& V_{, r}=e^{2 \beta}\left(1-4 \pi r^{2}(\rho h-2 p)\right) .
\end{aligned}
$$

Following [27] we express the hydrodynamic quantities on the right-hand side of Eqs. (23)-(24) solely in terms of the conserved hydrodynamic quantities U. This avoids additional iterations when using explicit algorithms to solve these ordinary differential equations.

In summary, the initial value problem consists of equations (4), (16), (20,22), (23,24), the scalar field initial data $\Phi\left(r, u_{0}\right)$ and initial and boundary data for the fluid variables $\left(\rho, \varepsilon, u^{r}\right)$ on the initial slice $\Sigma_{0}$ (at time $u_{0}$ ). Those equations and initial data are sufficient for obtaining a global solution to the problem.

\section{Global quantities}

Making use of the characteristic formulation of general relativity and covering the infinite range of the radial coordinate with a finite grid allows us to refer to some global quantities of the spacetime such as the Bondi mass and 
the news function. Apart from their physical relevance, these quantities can be used in global tests of our numerical evolutions, as we will show below.

Instead of extracting the Bondi mass directly at future null infinity we use the expression

$$
M=4 \pi \int_{0}^{\infty} r^{2} e^{-2 \beta} T_{r u} d r
$$

for the Bondi mass at time $u$ in our numerical implementation. Similarly, the news can be rewritten as 15]

$$
N=\frac{1}{2} e^{-2 H} \int_{0}^{\infty} \frac{V}{r} \Phi_{, r} d r .
$$

With these definitions, global energy conservation can be established,

$$
M(u)-M(0)=\int_{0}^{u}-4 \pi N(\hat{u})^{2} e^{2 H(\hat{u})} d \hat{u} .
$$

\section{NUMERICAL IMPLEMENTATION}

In order to study the interaction of the scalar field and the neutron star in a global spacetime we use nonequidistant grids for the radial coordinate $r$. Furthermore, to avoid dealing with complicated stencils in the numerical implementation, we make use of the following procedure, generalizing previous implementations in characteristic numerical relativity (see, e.g. [12]): Starting with an equidistant grid in the coordinate $x \in[0,1]$, we allow for a general coordinate transformation $r=r(x)$. Using the chain rule we rewrite the partial derivatives appearing in the above equation with

$$
()_{, r}=()_{, x} \frac{d x}{d r},
$$

thus effectively rewriting all our equations in the coordinate $x$. Unless otherwise stated we use the relation

$$
r=\frac{15 x}{1-x^{4}}
$$

for all computations presented in this work. Using such a coordinate transformation the repartition of grid points in the coordinate $r$ is almost equidistant for small radii and gets infinitely sparse for $x \rightarrow 1$, which corresponds to future null infinity $\mathcal{J}^{+}$.

We use a second order Runge Kutta method to solve the metric equations (23) and (24). To determine the equilibrium models of our relativistic stars, we also use the Runge Kutta method to solve the so-called TolmanOppenheimer-Volkoff equations, formulated on a null hypersurface as in [13].

The integration of the evolution equation for the scalar field, Eq. (16) (or equivalently Eq. (14)), proceeds with the specification of initial data $\Phi\left(u_{0}, r\right)$ on the initial null cone $u_{0}$. For the characteristic evolution we have used two different algorithms, both marching from the origin to the exterior.

The first procedure is based upon the construction of a null parallelogram built up from incoming and outgoing radial characteristics [28]. In this procedure one needs first to determine the right hand side of Eq. (16) at the center of the parallelogram to the desired order of accuracy. Then, an integral relation between this source term and the values of $g$ at the four corners of the parallelogram - which do not necessary have to coincide with grid points - has to be employed in order to compute the scalar field at that corner of the parallelogram lying next to the grid point which is to be updated. Suitable interpolations then give the scalar field at the new grid point to second order accuracy.

The second alternative procedure we have implemented to solve the scalar field equation is based on a direct discretization of Eq. (14) using a second order, finite difference, nondissipative algorithm discussed in [29].

Due to the stencils of both algorithms, we cannot use them at the origin, where a regular behavior of the scalar field as $\Phi=a+b r+c r^{2}$ is assumed. The linear term introduces a kink at the origin, but it is necessary in our foliation - as can be seen from the analytic solution for the wave equation in Minkowski space consisting of an ingoing and outgoing wave. Note that the scalar field enters the metric only through Eq. (23), thus respecting the regularity conditions given by Eqs. (7, ,8). Substituting this ansatz into Eq. (14) and grouping those terms with the same powers of $r$ we find that $a_{, u}=b, b_{, u}=1.5 c$. Extracting the coefficients $a, b$ and $c$ on the null cone $u_{0}$, we update $a$ and $b$ to obtain the scalar field at the first two grid points of the new hypersurface, which then allows us to start the marching procedure along the null hypersurface with either of the two algorithms described above.

By experimenting with both algorithms, we found that, on the one hand, the scheme based upon a direct discretization of the wave equation is more accurate in the long-term behavior in the interior of the numerical domain. This was relevant to resolve the late time fall-off behavior of the scalar field, as we describe below in Section $\mathrm{V}$. On the other hand, the algorithm based upon the null parallelogram is, however, superior close to future null infinity, where we regularized the equations following the work of [12]. Therefore, for the results presented in this work we have used a "hybrid algorithm", in which a direct discretization of Eq. (14) is used in the interior of the computational domain and the parallelogram algorithm is used close to future null infinity $\mathcal{J}^{+}$.

Concerning the numerical integration of the system of hydrodynamic equations, its hyperbolic mathematical character allows for a solution procedure based on the computation of (local) Riemann problems at each cellinterface of the numerical grid. At cell $i$ the state-vector $\mathbf{U}$ is updated in time (from $u^{n}$ to $u^{n+1}$ ) using a conservative algorithm 


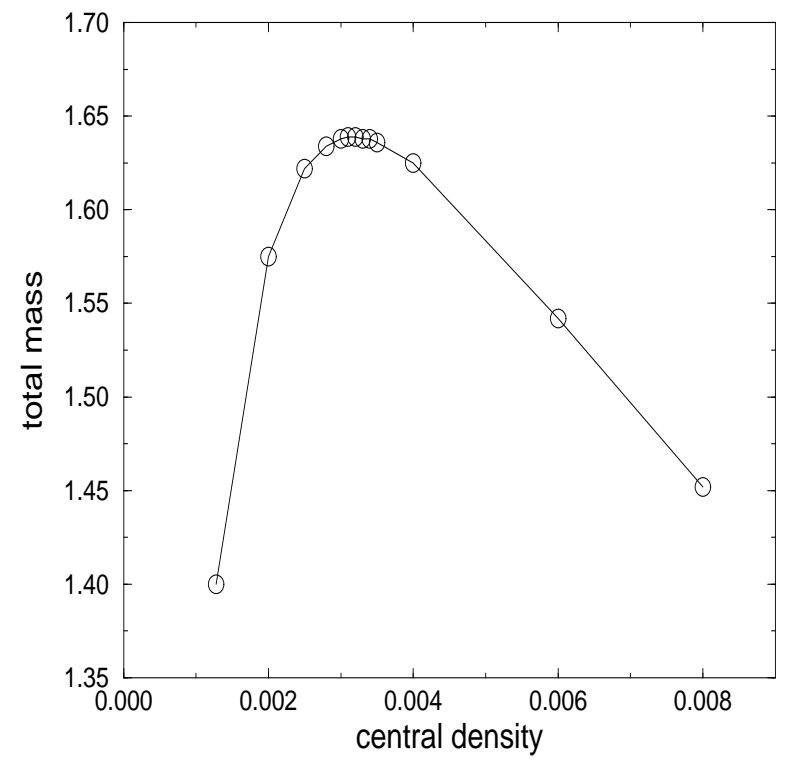

FIG. 1. Stability curve for neutron star models with the polytropic equation of state $p=K \rho^{\Gamma}$, with $K=100$ and $\Gamma=2$. Models lying to the left of the maximum of the curve, at about $\rho_{c}=3.2 \times 10^{-3}$, are stable against gravitational collapse. The circles are calculated with our initial data solver and are connected by straight lines. We use units in which $G=c=M_{\odot}=1$.

$$
\mathbf{U}_{i}^{n+1}=\mathbf{U}_{j}^{n}-\frac{\Delta u}{\Delta x}\left(\widehat{\mathbf{F}}_{i+1 / 2}-\widehat{\mathbf{F}}_{i-1 / 2}\right)+\Delta u \mathbf{S}_{j},
$$

where the numerical fluxes, $\widehat{\mathbf{F}}$, are evaluated at the cell interfaces according to some particular flux-formula which makes explicit use of the full spectral decomposition of the system. For our particular formulation of the hydrodynamic equations such characteristic information was presented in 13.

In more precise terms the hydrodynamics solver of our code uses a second order Godunov-type algorithm, based on piecewise linear reconstruction procedures at each cell-interface [30] and the HLLE approximate Riemann solver 31,32. General information on such schemes in relativistic hydrodynamics can be found, e.g. in [33] and references therein.

\section{CODE TESTS}

We now present representative results obtained in the process of code calibration. The assessment of the numerical implementation is provided by comparing to previous results and by checking global energy conservation tests.

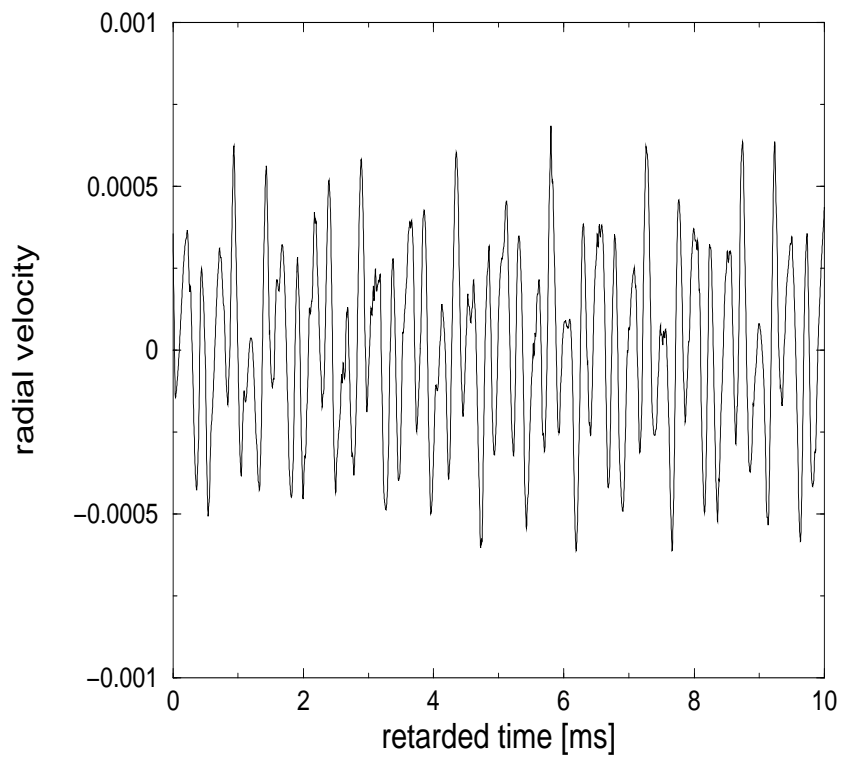

FIG. 2. Time evolution of the radial velocity $u^{x}$ at half radius of the star. The neutron star model has a central density $\rho_{c}=1.5 \times 10^{-3}$. The oscillations are essentially undamped for the evolution shown, which reflects the small viscosity of the hydrodynamic schemes employed.

\section{A. Null cone evolutions of self-gravitating, stable neutron stars}

As a first step to validate our numerical code we start by studying its ability to keep the equilibrium of initially stable neutron star models. For this purpose we perform long-term simulations of such initial data and analyze the stability of the code. Furthermore, we use these evolutions to compute the frequencies of the radial modes of pulsation. We compare the frequencies obtained with our nonlinear code to results of linear evolutions from perturbation theory.

For all simulations presented in this paper the neutron star models are approximated by a polytropic EoS, defined by $p=K \rho^{\Gamma}$, with polytropic constant $K=100$ and adiabatic exponent $\Gamma \equiv 1+1 / n=2$. Hence, the index of the polytrope is $n=1$. For the simulations presented in this section we choose two different models with central density $\rho_{c}=1.5 \times 10^{-3}$ and $\rho_{c}=2.8 \times 10^{-3}$ (recall that we are using units in which $M_{\odot}=1$ ). Both models are located in the stable branch of the central density - total mass - diagram (see Fig. 1).

When evolving these neutron star models with our numerical code we are able to maintain them in stable equilibrium for thousands of light-crossing times of the star without any sign of numerical instabilities.

To validate the code further we computed the frequencies of the radial modes of pulsation. For this aim we have to allow the star to (radially) contract and expand 


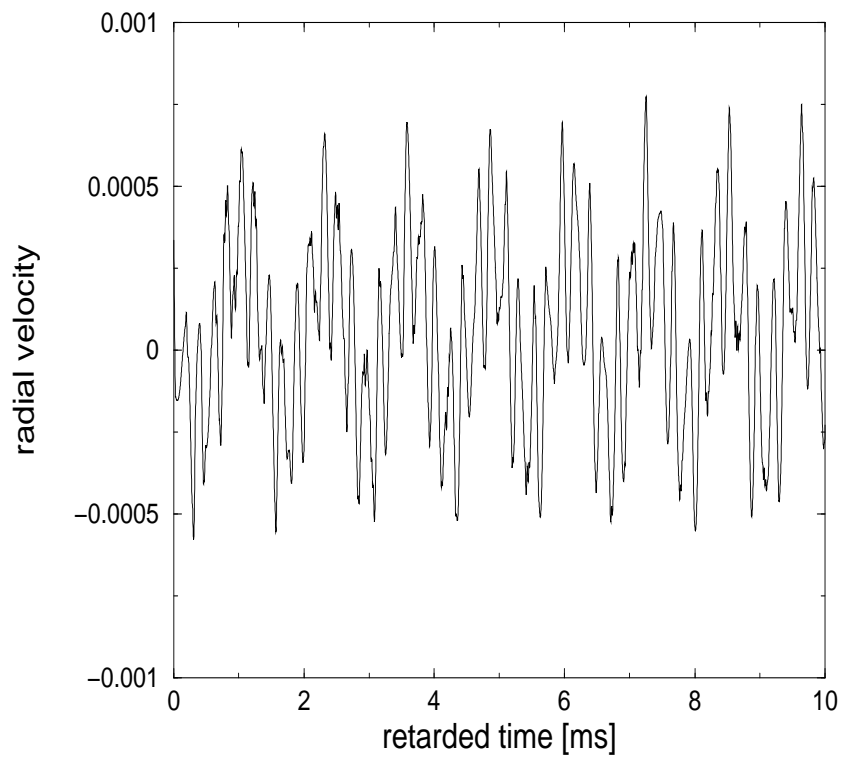

FIG. 3. Time evolution of the radial velocity $u^{x}$ at half radius of the star. The neutron star model has a central density $\rho_{c}=2.8 \times 10^{-3}$.

during the evolution. To this end, following 34] (see also [35), we surround the star with a few zones representing an artificial "atmosphere" filling an otherwise vacuum region. The density in this atmosphere is set to sufficiently small values such that its presence does not affect the dynamics of the system. The typical values we choose are $10^{-7}-10^{-8}$ times the central density of the star. Furthermore, to avoid any numerical problems due to (shock) heating in the atmosphere (the fluid in those zones is not in equilibrium and, therefore, it will collapse/accrete onto the neutron star), we follow the recipe described in [34] and enforce adiabatic evolution (by using the polytropic EoS) in the atmosphere and in the outer layers of the neutron star (comprising the outermost 10 grid points). After each time step, if the density has fallen below 1.5 times the density of the atmosphere, the hydrodynamic quantities are reset to their atmosphere values. The innermost location where this procedure is done defines the radius of the star. As described in more detail in the next section the above values of the atmosphere density are small enough to guarantee conservation of energy despite the artificial resetting procedure.

When evolving our stellar models in time, we find small deviations around the equilibrium values due to the discretization errors. As a result, the stars oscillate in their fundamental radial modes. Figures 2 and 3 show the radial velocity at half stellar radius for the above models as a function of the retarded time measured by distant observers. These simulations were performed with a grid of 800 zones covering the complete radial domain. This

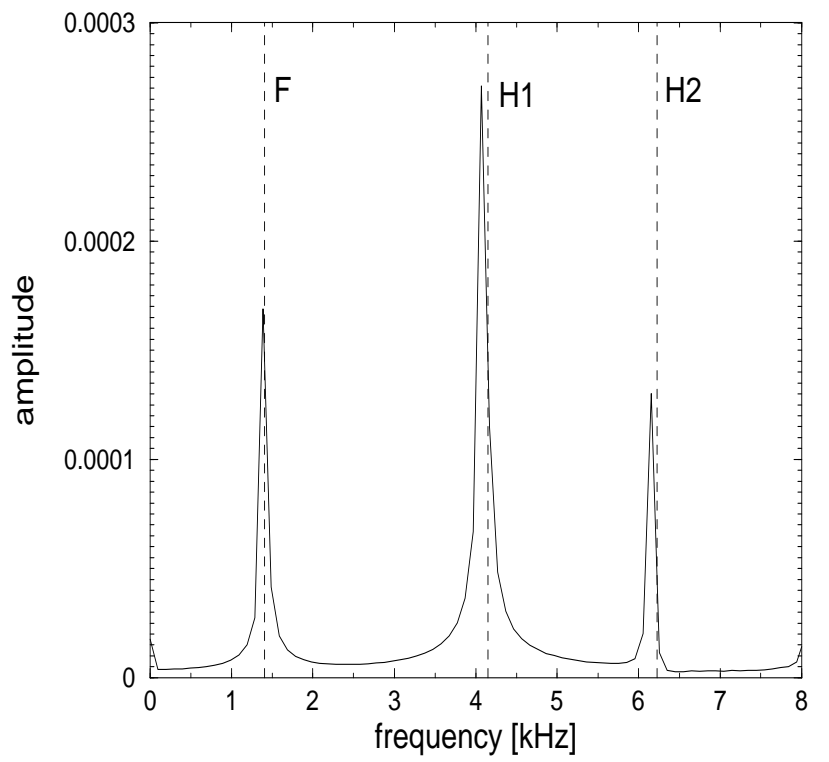

FIG. 4. Fourier transform of the time evolution shown in Fig. 2. The peaks in the Fourier transform indicate the mode frequencies of the fundamental radial mode (around $f=1.4 \mathrm{kHz}$ ) and the first two harmonics. The neutron star model has a central density $\rho_{c}=1.5 \times 10^{-3}$. The dashed vertical lines indicate the corresponding frequencies obtained with a perturbative (linear) code. The units in the $y$-axis are arbitrary.

amounts in using about half of the grid in resolving the neutron star (We choose this resolution here to allow for comparisons with the results of section $\mathrm{V}$, where we have to resolve the scalar field as well.) As shown in 335] one can use such evolutions to obtain the frequencies of the excited modes of pulsation of the star by simply Fourier transforming those data. In general, however, the excitation of the different modes by the truncation error of the numerical schemes may not be sufficient to accurately determine the mode frequencies. Therefore, in order to compare unambiguously our mode frequencies with perturbation theory, we further perturb the density of the equilibrium models with an explicit eigenfunction $\rho=\rho_{o}+A \rho_{c} \sin (\pi r / R)$, where $R$ denotes the radius of the star (see Table I) and $\rho_{o}$ is the density of the unperturbed star. The typical amplitude we use in the perturbation is $A=10^{-6}$.

Figures 1 and 5 show the frequencies of the fundamental mode and the first two overtones obtained by a Fourier transform of the radial velocity evolutions. The dashed vertical lines in these plots were obtained using a linear code [36] which evolves in time the perturbation equations. The agreement between the two codes is remarkable. The fundamental mode of the model with $\rho_{c}=2.8 \times 10^{-3}$ is already rather small, the star being close to the unstable branch. We note that the code is 


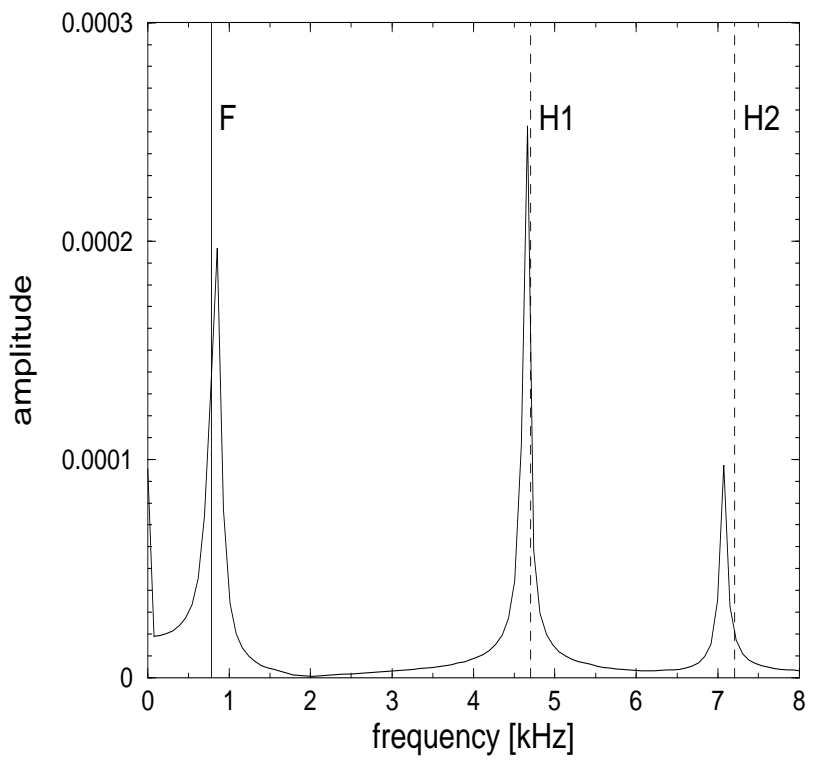

FIG. 5. Fourier transform of the time evolution shown in Fig. 3. The frequencies of the fundamental radial mode (around $f=0.8 \mathrm{kHz}$ ) and the first two overtones are shown for a neutron star with central density $\rho_{c}=2.8 \times 10^{-3}$.

able to obtain a much higher number of overtones. Nevertheless, for the sake of clarity in the comparison and for our purpose of assessing the correct numerical implementation, it is sufficient to show only the first two harmonics.

\section{B. Scalar field dynamics in a regular spacetime}

In this section we present results aimed to validate the numerical implementation of the Einstein-Klein-Gordon solver. For this purpose we investigate the reflection of a scalar field at the origin of the coordinate system, turning off the hydrodynamics module of the code. The initial data for the scalar field packet are

$$
\Phi_{0}=2 \times 10^{-3} e^{-(r-14)^{2}} .
$$

The location of this Gaussian pulse is chosen in such a way that, if superposed on the neutron star spacetimes of the previous section, the scalar wave pulse would initially lie outside the neutron star, the overlap being completely negligible. Evolving this data, the initial pulse approaches the origin, is reflected, and radiates away, leaving behind Minkowski space. Such a sequence can be followed in Fig. 6, for a simulation employing a grid of 800 zones. We note the stability and smoothness of the solution, both at the origin and at $\mathcal{J}^{+}$.

By evaluating global energy conservation, according to Eq. (27), after the pulse has reflected off the origin, we

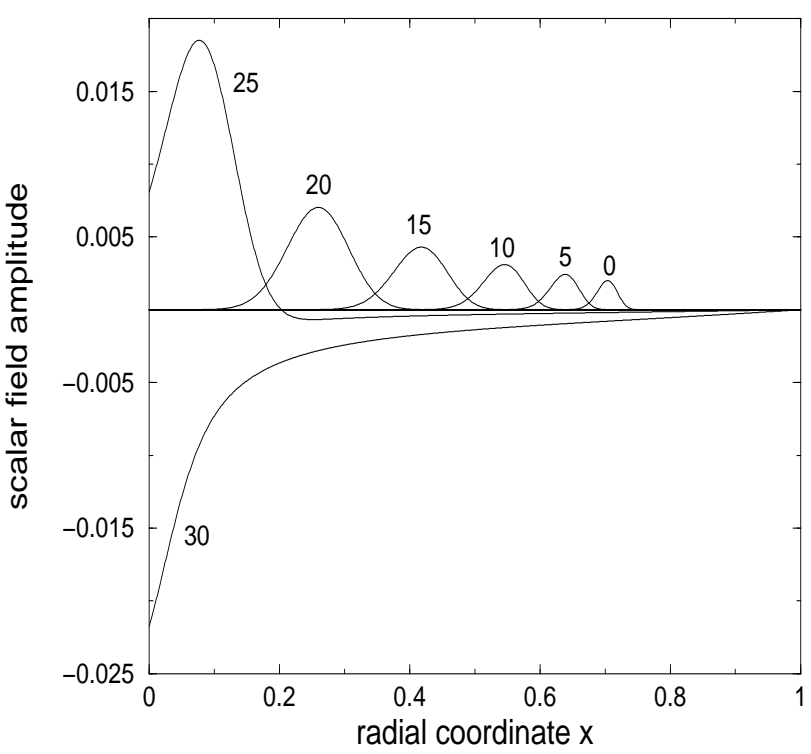

FIG. 6. Radial profiles of the scattering of a scalar field off the origin of the coordinate system at different times of its evolution. The numbers in the plot refer to the time coordinate $u$. For times $u \geq 35$ the pulse has completely radiated away leaving Minkowski spacetime behind.

find that the energy is conserved (as expected) to second order accuracy. The best linear fit to the curve shown in Fig. 7 gives a slope of 1.99 .

As an aside we note that by simply changing the origin treatment in the code, it is possible to study the evolution of a scalar field outside a spherical black hole. We performed such a simulation finding agreement with the results of [19].

\section{Scalar field in a dynamic spacetime with a neutron star}

We consider now the full set of equations and prescribe initial data consisting of the ingoing scalar field pulse given by Eq. (31), together with a stable equilibrium, self-gravitating neutron star model with initial central density $\rho_{c}=1.28 \times 10^{-3}, K=100$ and $\Gamma=2$. This relativistic star model has a total mass of $1.4 M_{\odot}$ (see also [35]). We perform simulations of the scalar field scattering off the neutron star, focusing our study in this Section on the assessment of the global energy conservation properties of our complete numerical implementation. A comprehensive study of the dynamics of the scattering is deferred to Section $\mathrm{V}$.

Fig. 8 shows the Bondi mass of the neutron star - scalar field spacetime as a function of retarded time, combined with the total mass of the scalar field radiated away to null infinity. As one can clearly see from this figure, the 


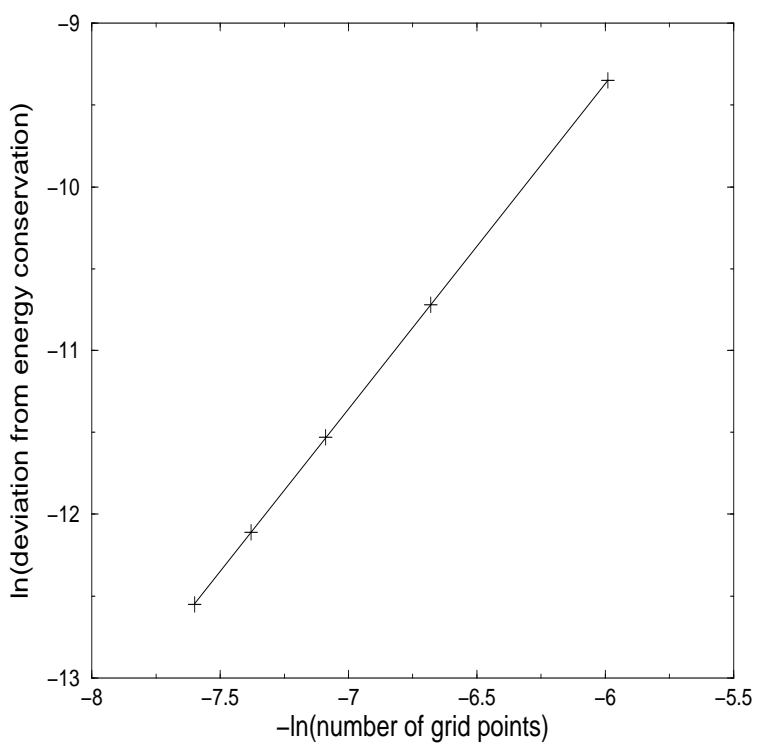

FIG. 7. Convergence test of the global energy conservation for the Einstein-Klein-Gordon system once the scalar wave has completely radiated away after reflecting off the origin. The best linear fit to our results has a slope of 1.99, confirming the (global) second order accuracy of our numerical implementation.

spacetime is losing mass exactly at the rate which is radiated to null infinity by the scalar field.

By computing Eq. (27) at a fixed retarded time of $\tau=0.5 \mathrm{~ms}$ for different grid resolutions, we find that our code conserves globally the energy with a convergence rate which lies in between 1 and 2 . The best linear fit is depicted in Fig. 9. The fact that the convergence rate drops now below second order is, however, to be expected, since the approximate Riemann solver we are using for the integration of the hydrodynamic equations is only (locally) first order accurate at discontinuities (i.e., the surface of the star) and at local extrema (i.e., the center of the star) (see the related discussion in [35]). Nevertheless, for the highest resolution we have used, 2000 radial grid points, the relative error in the energy conservation is of the order of $2 \times 10^{-6}$ for this very dynamical simulation.

\section{DYNAMICS OF SCALAR FIELD - NEUTRON STAR INTERACTIONS}

In this section we present our main results concerning the scattering of a scalar field pulse off relativistic neutron stars. As mentioned before, we use $n=1$ relativistic polytropes as neutron star models. All models we construct lie on the stable branch of the total mass-central density diagram (see Fig. 11) and are characterized by increasing central densities and compactness. Their basic properties are summarized in Table $\mathbb{\text { If }}$.

In addition to the neutron stars we construct a Gaus-

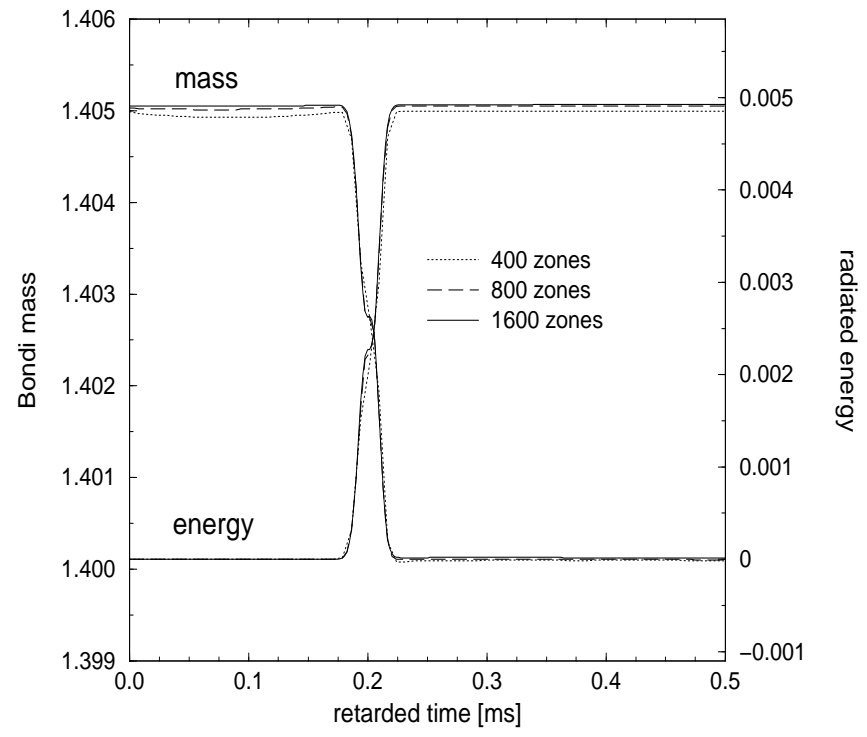

FIG. 8. Bondi mass of the spacetime and total radiated energy of the scalar field as a function of retarded time for different resolutions. At the beginning, the scalar field contributes to the Bondi mass of the spacetime $\left(1.405 M_{\odot}\right)$, before the Bondi mass drops in a small time interval, when the main part of scalar field mass (about $5 \times 10^{-3} M_{\odot}$ ) is emitted to future null infinity $\mathcal{J}^{+}$. As the sum of the two curves is constant, the energy is globally conserved (with a relative error of about $5 \times 10^{-6}$ for the run with 1600 zones.)

sian pulse of a scalar field according to Eq. (31), thus fixing the pulse amplitude, width and location. For these initial data there is no overlap between the star and the scalar field pulse which then makes it possible to associate a specific initial mass with each one of the matter fields.

In our setup we keep the central density as the only free parameter of the simulations, choosing a unique polytropic EoS and fixing the geometry and amplitude of the scalar field. This is clearly a severe restriction in the parameter space of the scattering problem. Nevertheless, we choose this particular setup since we are interested in investigating the relativistic effects of the interaction, where the scalar field has a strong impact on the dynam-

TABLE I. Equilibrium properties of the $K=100, n=1$ neutron star models in units in which $c=G=M_{\odot}=1$. The entries are as follows: $\rho_{c}$ is the central density, $M$ and $R$ are the mass and radius of the star, respectively, and $C=2 M / R$ is the compactness parameter.

\begin{tabular}{cccc}
\hline \hline$\rho_{c}\left(10^{-3}\right)$ & $M$ & $R$ & $C=2 M / R$ \\
\hline 1.5 & 1.47 & 9.26 & 0.317 \\
2.2 & 1.60 & 8.45 & 0.379 \\
2.8 & 1.63 & 7.91 & 0.412 \\
2.9 & 1.64 & 7.84 & 0.418 \\
3.0 & 1.64 & 7.76 & 0.423 \\
\hline \hline
\end{tabular}




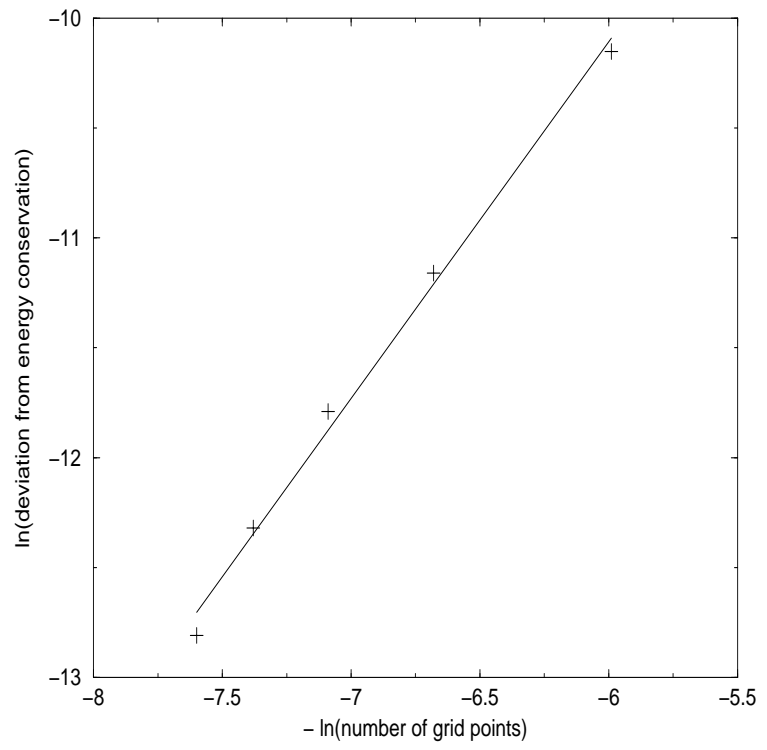

FIG. 9. Convergence test of the global energy conservation of a dynamic spacetime containing a self-gravitating neutron star and a scalar field. The rate of convergence is 1.62 . See text for details.

ics of the neutron star. A detailed analysis of the whole parameter space is beyond the scope of this work.

When evolving in time the initial data, the scalar field travels inwards, enters the neutron star and it is finally reflected at the origin of the coordinate system. Contrary to the Einstein-Klein-Gordon system without the perfect fluid, which was discussed in Section IVB, the presence of the neutron star, and its associated potential well, may give rise to a phase of multiple interactions of the wave back and forth the origin and the maximum of the curvature potential. This, in turn, reflects itself in the existence of quasi-periodic signals (trapped modes), as discussed by [26] before its energy is radiated away. Furthermore, our neutron star models have been chosen conveniently close to the maximum of the stability curve (see Fig. 1). Depending on the compactness of the neutron star onto which the wave pulse impacts, the stars are forced to either oscillate violently, or to collapse to a black hole on a dynamical timescale. Fig. 10 shows the spacetime diagram for the least compact neutron star model of our sample, with $\rho_{c}=1.5 \times 10^{-3}$. For this model, the scalar field is able to force the star to contract and to expand, pulsating radially, as can clearly be identified in the varying location of the star's radius (the vertical solid line in Fig. 10). With our foliation, an outgoing scalar wave is covered in only one slice.

Fig. 11 displays the time evolution of the central density of the different neutron stars in our setup. The solid lines correspond to the neutron star-scalar field system. Correspondingly, the dashed horizontal lines indicate the evolutions of the equilibrium neutron star models without the presence of the scalar field. As already mentioned these evolutions are stable. They are characterized by

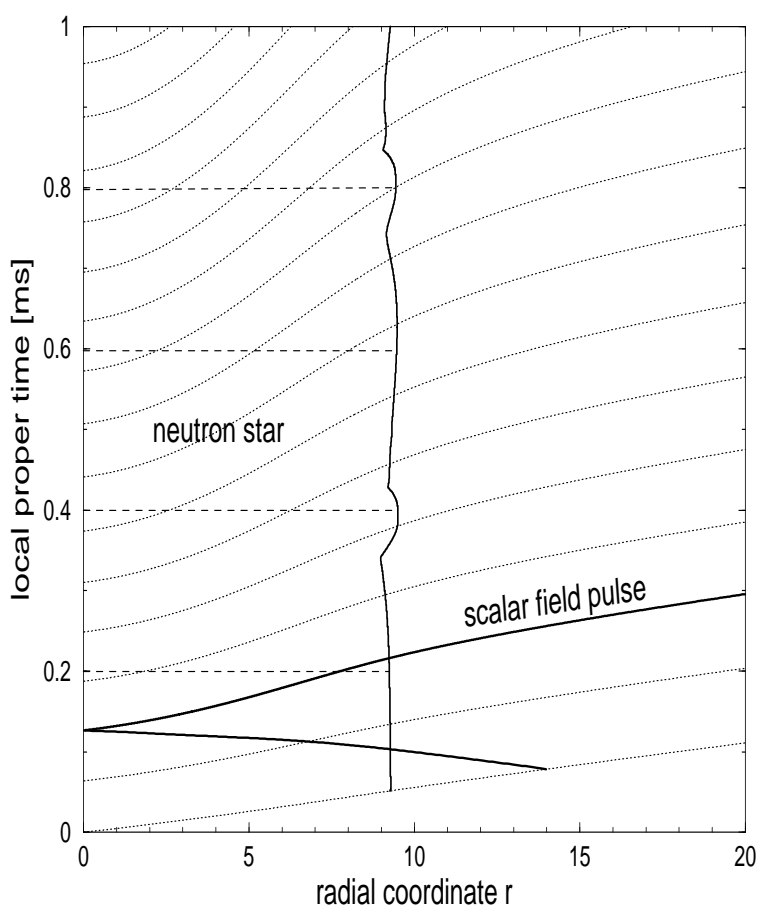

FIG. 10. Spacetime diagram of the reflection of a Gaussian scalar field pulse off a neutron star $(K=100, n=1$ and $\left.\rho_{c}=1.5 \times 10^{-3}\right)$. The diagram focuses in the region close to the neutron star - scalar field interaction, but it was obtained from a global simulation of the spacetime. The dotted curves covering the whole diagram are outgoing light cones, which bend due to the spacetime curvature. The scalar field pulse, initially located at $r=14$, travels inwards, enters the neutron star and is reflected at the origin of the coordinate system (the solid line corresponds to the maximum value of the scalar field). The interaction with the scalar field triggers the oscillation of the neutron star, which can be seen from the vertical solid line of varying location in the diagram, which indicates the radius of the star.

the appearance of small-amplitude oscillations associated with the radial modes of pulsation of the star (which are too small to be seen in the figure). On the other hand, all neutron star-scalar field models with initial central density below $2.8 \times 10^{-3}$ also oscillate around the stable equilibrium model. The oscillation frequencies of the two least compact models correspond to the frequencies calculated in the linear regime, even though the amplitude of the oscillations is now much larger due to the scalar field kick. This is no longer the case for the model with a central density of $\rho_{c}=2.8 \times 10^{-3}$. For this model, which is close to the threshold of black hole formation, the amplitude of the oscillations is big enough to show nonlinear effects, the oscillation frequency being much smaller than the value obtained in the previous section. For the models with central densities of $\rho_{c}=2.9 \times 10^{-3}$ and $\rho_{c}=3.0 \times 10^{-3}$ the interaction with the scalar field is able to trigger their gravitational collapse to a black hole on a dynamical timescale. Unfortunately, due to nu- 


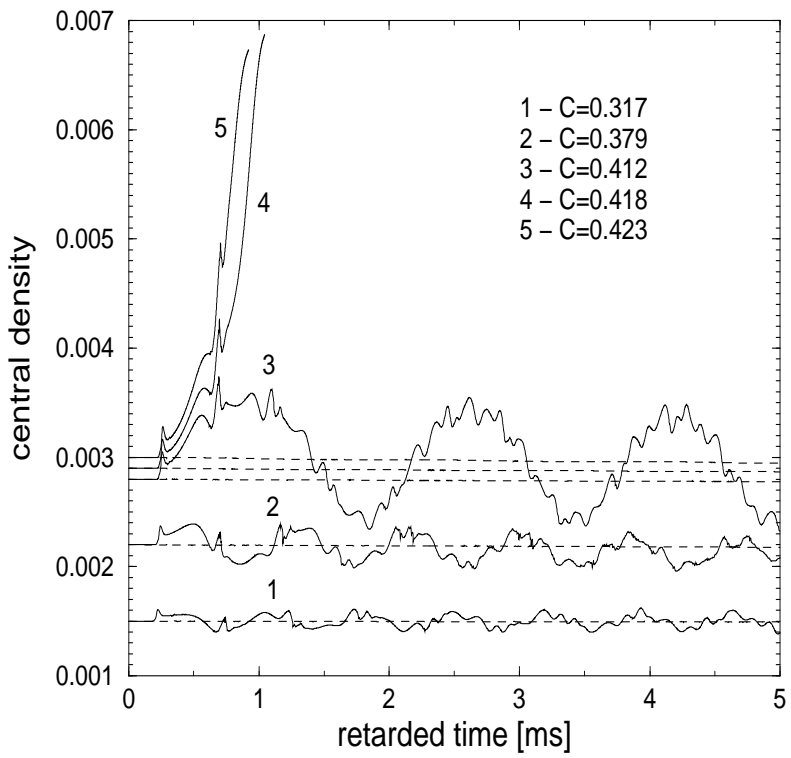

FIG. 11. Central density of the neutron stars interacting with the scalar field as a function of retarded time. The three less compact models, with $\rho_{c} \leq 2.8 \times 10^{-3}$, oscillate strongly around their equilibrium value after interacting with the scalar field. The other two more compact models collapse to a black hole instead on a dynamical timescale. The dashed lines are taken from our evolutions of the equilibrium model without the presence of the scalar field.

merical inaccuracies arising at the end of the simulation we are not able to follow the collapse process once the event horizon is about to form (similar problems were reported in 27] for the collapse of supermassive stars). However, convergence studies show clear evidence that these models collapse to black holes. Further evidence is given by the evolution of the neutron star radii, as shown in Fig. 12 .

To demonstrate the dynamic range of these evolutions, we focus on the model with central density $\rho_{c}=2.9 \times$ $10^{-3}$. Initially, the redshift factor $e^{2 H}$ between the center of the star and observers located at $r \rightarrow \infty$ is 2.1. By the end of the simulation it has increased to a value of 59.5. This high redshift factor explains the appearance of a kink in the central density towards the end of our numerical evolution (see Fig. 11).

We also note that global energy conservation is very well fulfilled for these extreme hydrodynamic simulations. The relative deviation from energy conservation according to Eq. (27) when the evolution stops is of the order of $10^{-4}$.

By analyzing the energy transfer from the scalar field to the neutron star during the interaction we find that it increases the more compact the stellar models are. This behavior is shown in Table III. We remark that the initial mass of the scalar field is not strictly identical for all cases

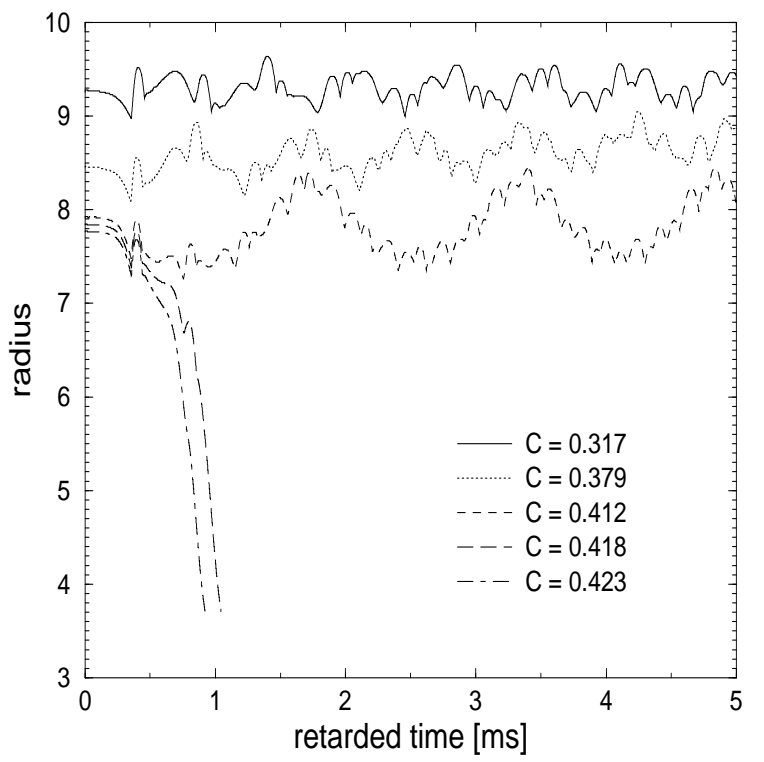

FIG. 12. Time evolution of the radius of the different neutron stars interacting with the scalar field. The radius of two most compact models decreases dramatically, indicating that they undergo gravitational collapse to a black hole.

considered, due to the different underlying geometry on which the Gaussian pulse is constructed. We evaluate the total radiated mass in the scalar field at a retarded time of $\tau=0.6 \mathrm{~ms}$. The mass radiated away to infinity after this time is negligible.

Next we analyze the behavior of the scalar field in these scattering simulations. In Fig. 13 we plot the (retarded) time evolution of the news, Eq. (26), for the whole sample of our neutron star models. The scalar field signal measured at null infinity can be neatly divided into three phases. The first phase, before the main pulse reflects off the origin (not shown in the figure), is dominated by an initial backscattering, the amplitude of the signal being small. The second phase, whose duration depends on the compactness of the neutron star [26], is characterized

TABLE II. Energy transfer from the scalar field to the neutron star during the scattering process. The entries are as follows: $\rho_{c}$ is the central density of the neutron star, $M_{0}^{\Phi}$ is the initial mass of the scalar field, $E_{\text {rad }}$ is the total radiated mass, and $E_{\text {trans }}$ is the percentage of the energy transfered in the interaction. We use units in which $G=c=M_{\odot}=1$.

\begin{tabular}{cccc}
\hline \hline$\rho_{c}\left(10^{-3}\right)$ & $M_{0}^{\Phi}\left(10^{-3}\right)$ & $E_{\text {rad }}\left(10^{-3}\right)$ & $E_{\text {trans }}(\%)$ \\
\hline 1.5 & 4.90 & 4.86 & 0.8 \\
2.2 & 4.80 & 4.72 & 1.7 \\
2.8 & 4.76 & 4.65 & 2.3 \\
2.9 & 4.75 & 4.63 & 2.5 \\
3.0 & 4.75 & 4.62 & 2.7 \\
\hline \hline
\end{tabular}


by the reflection of the main scalar field pulse back and forth the origin and the maximum of the neutron star curvature potential, which, in turn, induces the appearance of quasi-normal oscillations on the scalar field. Most of the energy is radiated away in this period. Once the pulse has lost sufficient energy it enters a third phase, in which the late time behavior of the signal is dominated by a power-law tail $N \propto t^{-\alpha}$, with $\alpha=3$, due to the reflection of the scalar field at the exterior Schwarzschild geometry [21, 17, 18, 26]. Since the compactness of our models is well below the Buchdahl limit, $C=8 / 9$, the quasi-normal mode ringing phase does not last for an extended period of time. Therefore, after a few reflections trapped inside the curvature potential, the signal enters rapidly the power-law tail phase. From Fig. 13 one can see that the more compact the neutron star, the larger the quasi-normal ringdown phase. We also point out that by going to more compact models, increasing the central density of the neutron star beyond the maximum of the stability curve (i.e., going into the unstable branch) and freezing the hydrodynamics and metric evolution to avoid gravitational collapse, we are able to find a much longer ringdown phase. Our results, obtained for fully self-gravitating, polytropic neutron star models, are in perfect agreement with previous findings by Pavlidou et al [26], who used a more idealized setup consisting of constant density, static neutron stars.

Since the study of the late time power-law tails requires sufficient resolution, especially for large radii, we have used a different radial coordinate for these simulations, $r=30 x /\left(1-x^{4}\right)$. This allowed us to resolve the power-law behavior, avoiding the evolution from being dominated by numerical noise. By performing a linear regression study of the tails in the time interval $\log (\tau[m s]) \in[0.3 ; 0.7]$, we obtain the results summarized in Table III. We find the correct power-law behavior of the scalar field in our fully dynamical evolutions, as predicted by both, linear analysis and by nonlinear numerical evolutions of scalar fields in the exterior black hole geometry [17 19]. Note that we measure the tails on the news, whereas the results of the above references read off the quantity $g$ at future null infinity $\mathcal{J}^{+}$. Both quantities are related by

$$
N=e^{-2 H} g_{, u} .
$$

\section{SUMMARY}

We have analyzed numerically the interaction of neutron stars and scalar fields by means of nonlinear evolutions of the Einstein-Klein-Gordon perfect fluid system in spherical symmetry. We have built a sequence of stable, self-gravitating, $K=100, n=1$ relativistic polytropes, increasing the central density from $\rho_{c}=1.5 \times 10^{-3}$ to $3.0 \times 10^{-3}\left(G=c=M_{\odot}=1\right)$. Using a compactified spacetime foliation with outgoing null cones we have

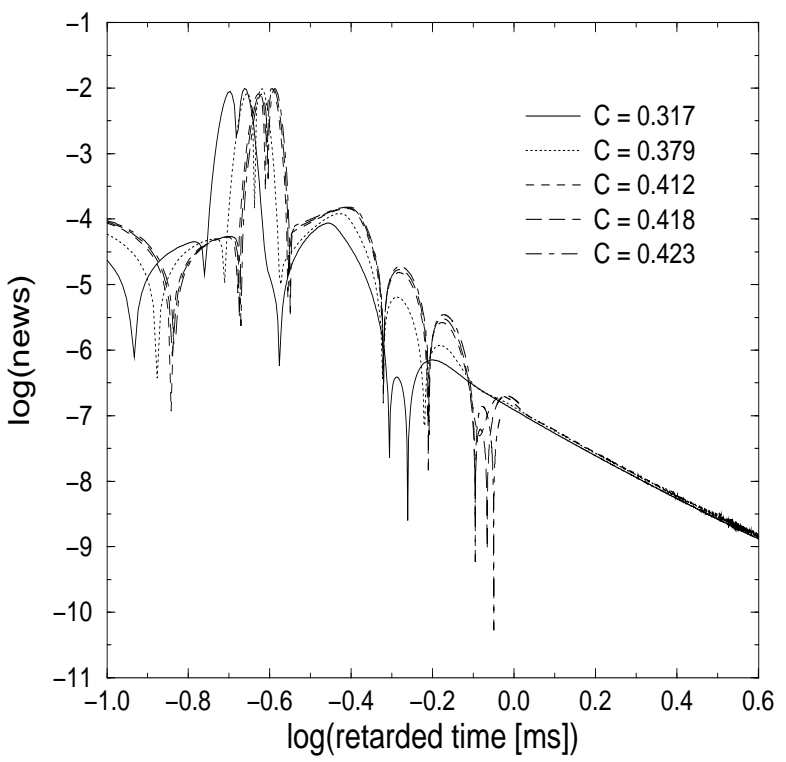

FIG. 13. Time evolution of the news function during the scattering problem. The different lines correspond to the dif-

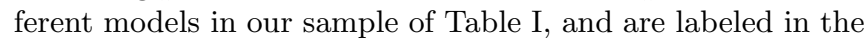
plot with respect to the compactness parameter. The duration of the more dynamic quasi-normal ringing phase strongly depends on the compactness of the neutron star model, increasing as the compactness increases. The late time behavior of the signal decays as an inverse power-law.

studied the fate of the neutron stars when they are hit by a sufficiently strong scalar field packet, as well as the dynamics and energetics of the process.

We have found that by choosing a strong (finite amplitude) scalar field pulse with a mass of the order of $10^{-3} M_{\odot}$, the neutron star is either forced to oscillate in its radial modes of pulsations or to collapse to a black hole on a dynamical timescale. The fate of the star depends on its central density and, since we fix the polytropic equation of state, on its compactness. The energy transfered to the neutron star increases with the compactness of the model. The radiative signals we have found in our fully nonlinear simulations consist of sev-

TABLE III. Late time power-law behavior of the news $N \propto t^{-\alpha}$ for the (stable) neutron star - scalar field scattering problem. The results agree with the predicted exact value $\alpha=3$.

\begin{tabular}{cc}
\hline \hline$\rho_{c}\left(10^{-3}\right)$ & $\alpha$ \\
\hline 1.5 & 3.06 \\
2.2 & 3.05 \\
2.8 & 3.05 \\
\hline \hline
\end{tabular}


eral quasi-normal oscillations and a late time power-law tail, in agreement with the results predicted by (linear) perturbation analysis of wave propagation in an exterior Schwarzschild geometry 21.

\section{ACKNOWLEDGEMENTS}

It is a pleasure to thank Ewald Müller for useful discussions and for a careful reading of the manuscript, and Johannes Ruoff by kindly providing us the frequencies of the fundamental modes of the neutron star models of Section IV A, obtained with his perturbation code. We further thank Nigel Bishop and Carsten Gundlach for helpful comments on the manuscript. F.S. would like to thank the Relativity and Cosmology Group of the University of Portsmouth, where part of this work was done. This work was supported, in part, by the EU Programme "Improving the Human Research and the Socio-Economic Knowledge Base" (Research Training Network Contract HPRN-CT-2000-00137). P.P. also acknowledges support from the Nuffield Foundation (award NAL/00405/G).

[1] C.L. Fryer, D.E. Holz and S.A. Hughes, astro$\mathrm{ph} / 0106113$

[2] R.F. Stark and T. Piran, Phys. Rev. Lett., 55, 891 (1985)

[3] M. Shibata, Prog. Theor. Phys., 104, 325 (2000).

[4] H. Dimmelmeier, J.A. Font and E. Müller, astro$\mathrm{ph} / 0103088$.

[5] H.-Th. Janka, K. Kifonidis and M. Rampp, Proc. Workshop on Physics of Neutron Star Interiors, Ed. D. Blaschke, N.K. Glendenning, A.D. Sedrakian, Springer, in press, (2001) astro-ph/0103015.

[6] E. Müller, in Computational methods for astrophysical fluid flow, O. Steiner and A. Gautschy Eds., Springer Verlag, Berlin, 343-494 (1998).

[7] H. Bondi, M.G.J. van der Burg, A.W.K. Metzner, Proc. Roy. Soc. A, 269 21-52 (1962).

[8] R.K. Sachs, Proc. R. Soc. A 270 103-126 (1962).

[9] L.A. Tamburino, J.H. Winicour, Phys. Rev. 150 10391053 (1966).

[10] R.A. Isaacson, J.S. Welling, and J. Winicour, J. Math. Phys., 24, 1824 (1983).

[11] J. Winicour, Living Rev. Relativ., 4, 3 (2001).

[12] R. Gómez, P. Papadopoulos, J. Winicour, J. Math. Phys. 35(8) 4184-4204 (1994).

[13] P. Papadopoulos, J.A. Font, Phys. Rev. D 61024015 (2000).

[14] P. Papadopoulos, J.A. Font, Phys.Rev. D 63044016 (2001).

[15] R. Gómez, J. Winicour, Journ. Math. Phys. 33 1445-1457 (1992).

[16] R.L. Marsa, M.W. Choptuik, Phys. Rev. D 54 4929-4943 (1996).

[17] C. Gundlach, R.H. Price, J. Pullin, Phys. Rev. D 49(2) 883-889 (1994).
[18] C. Gundlach, R.H. Price, J. Pullin, Phys. Rev. D 49(2) 890-899 (1994).

[19] R. Gómez, J. Winicour, B. Schmidt, Phys. Rev. D 49(6) 2828-2836 (1994).

[20] P. Papadopoulos, P. Laguna, Phys. Rev. D 55 2038-2043 (1997).

[21] R.H. Price, Phys. Rev. D 5, 2419 (1972); 5, 2439 (1972).

[22] S.H. Hawley, M. Choptuik, Phys. Rev. D 62104024 (2000).

[23] K.D. Kokkotas, B.G. Schmidt, Living Rev. Rel. 2, 2 (1999).

[24] G. Allen, N. Andersson, K.D. Kokkotas, B.F. Schutz, Phys. Rev. D 58124012 (1998).

[25] J. Ruoff, Phys. Rev. D 63, 064018 (2001).

[26] V. Pavlidou, K. Tassis, T.W. Baumgarte, S.L. Shapiro, Phys. Rev. D 62084020 (2000).

[27] F. Linke, J.A. Font, H.T. Janka, E. Müller, P. Papadopoulos, Astron. Astrophys., in press (2001), astro$\mathrm{ph} / 0103144$.

[28] R. Gómez, J. Winicour, R. Isaacson, J. Comp. Phys. 98 11-25 (1992).

[29] L. Lehner, Journ. of Comp. Phys. 149 59-74 (1999).

[30] B. van Leer, J. Comput. Phys. 32101 (1979).

[31] A. Harten, P.D. Lax, B. van Leer, SIAM Review 25, 35 (1983).

[32] B. Einfeldt, SIAM J. Num. Anal. 25294 (1988).

[33] J.A. Font, Living Rev. Relativ. 3, 2 (2000).

[34] J.A. Font, M. Miller, W.-M. Suen, M. Tobias, Phys. Rev. D 61044011 (2000).

[35] J.A. Font, N. Stergioulas, K.D. Kokkotas, Mon. Not. R. Astron. Soc. 313 678-688 (2000).

[36] J. Ruoff, Private communication. 